\title{
Targeting family functioning, acculturative stress, and sugar-sweetened beverage consumption for obesity prevention: findings from the Hispanic community children's health study/study of Latino youth
}

Roger Figueroa ${ }^{1 *}\left(\mathbb{D}\right.$, Carmen R. Isasi ${ }^{2}$, Krista M. Perreira ${ }^{3}$, Amanda C. McClain ${ }^{4}$, Linda C. Gallo ${ }^{5}$, Daniela Sotres-Alvarez ${ }^{6}$, Alan M. Delamater ${ }^{7}$, Martha Daviglus ${ }^{8}$, Linda Van Horn ${ }^{8}$ and Josiemer Mattei ${ }^{9}$

\begin{abstract}
Background: Maintaining a bond with one's family as well coping with stress while acculturating to the US may protect Hispanic/Latino youth from increased sugar-sweetened beverages (SSB) consumption, which heightens the risk for overweight and obesity. This study aims to examine associations between acculturative stress, family functioning, and SSB consumption by acculturation status among U.S. Hispanic/Latino youth.

Methods: With cross-sectional data on 1465 youth 8-16y (49.6\% females) participating in the Hispanic Community Children's Health Study/Study of Latino Youth, we classified youths into four acculturation groups - assimilated, integrated, marginalized/separated, and unclassified. SSB consumption was assessed through two 24-h diet recalls and defined as intake frequency of soda, fruit juice, sweetened soft and fruit drinks. Multi-group path regression models were used to test associations of Hispanic/Latino youth' acculturative stress and family functioning with SSB consumption, as well as the moderating role of acculturation status.
\end{abstract}

Results: When controlling for age, sex, and study site, acculturative stress $(\beta=-0.13, p=0.01)$ was inversely associated with SSB, and poor family functioning $(\beta=0.11, p=0.07)$ was only marginally associated with SSB consumption among youth classified as assimilated but not among youth classified as integrated, marginalized/ separated, or unclassified.

Conclusions: A socio-ecological perspective that incorporates the role of key acculturation-related factors across multiple levels may aid efforts to identify mechanisms that influence the relationship between acculturation status and diet among Hispanic/Latino youth and their families.

Keywords: Hispanic/Latino, Acculturation, Adolescence, Sugar-sweetened beverages (SSB) consumption, Family functioning

\footnotetext{
* Correspondence: rf453@cornell.edu

'Division of Nutritional Sciences, College of Human Ecology, Cornell University, Ithaca, NY, USA

Full list of author information is available at the end of the article
}

C C The Author(s). 2020 Open Access This article is licensed under a Creative Commons Attribution 4.0 International License, which permits use, sharing, adaptation, distribution and reproduction in any medium or format, as long as you give appropriate credit to the original author(s) and the source, provide a link to the Creative Commons licence, and indicate if changes were made. The images or other third party material in this article are included in the article's Creative Commons licence, unless indicated otherwise in a credit line to the material. If material is not included in the article's Creative Commons licence and your intended use is not permitted by statutory regulation or exceeds the permitted use, you will need to obtain permission directly from the copyright holder. To view a copy of this licence, visit http://creativecommons.org/licenses/by/4.0/. The Creative Commons Public Domain Dedication waiver (http://creativecommons.org/publicdomain/zero/1.0/) applies to the data made available in this article, unless otherwise stated in a credit line to the data. 


\section{Background}

Global consumption of added sugars are the main source of empty calories contributing to daily energy intake, obesity, and diabetes [1-9], which is alarmingly prevalent in children's and youth' diets [10]. A popular form of added sugar intake in this age group, sugarsweetened beverages (SSB), substantially contributes to daily energy intake [10]. SSB include soft drinks (i.e., soda, fruit punches, lemonades), fruit juices and drinks, sports drinks, as well as energy drinks [11]. The major source of added sugar from SSB is $100 \%$ fruit juice, while both $100 \%$ fruit juice and soft drinks contribute similarly to total energy intake [12]. Large amounts of added sugars in SSB may also increase the risk of obesity by contributing to a high glycemic load and exacerbating insulin response [13]. SSB consumption, along with the daily calories sold per capita per day from all SSB, is highest in both North and Latin America [4], and is prevalent among Hispanic/Latino children and youth in Latin America and the United States (U.S.) [14, 15]. These numbers have pronounced implications for native and immigrant youth of Hispanic/Latino heritage living in the U.S., as they are disproportionally affected by obesity [16].

Existing disparities in SSB consumption and health outcomes such as obesity among Hispanic/Latino youth and their families living in the US may be associated with adoption of US customs and behaviors, versus maintenance of Latin American customs and behaviors [17]. Acculturation, broadly defined as the multidimensional process through which individuals economically, psychologically, and socio-culturally adapt from one cultural context to another due to migration or other life experiences $[18,19]$, has been associated with dietary behaviors and other obesity-related behaviors among Hispanic/Latino youth [20]. Research suggests that greater language-based acculturation to the U.S. among Hispanic/ Latino families is associated with negative health outcomes and less healthful dietary behaviors [17], including greater SSB consumption than their U.S.-born nonHispanic white counterparts [21]. Recently, Arandia et al. (2018) examined associations between a multidimensional measure of acculturation, the Acculturation Habits, and Interests Multicultural Scale for Adolescents (AHIMSA), and Hispanic/Latino youth's eating behaviors [22]. They found no differences in consumption of empty calories by acculturation group [22]. However, the study did not specifically examine SSB consumption [23].

Because acculturation tends to occur more rapidly among Hispanic/Latino youth than among their parents [24], understanding the role of acculturation on SSB consumption patterns in youth is essential. One plausible factor influencing SSB consumption through acculturation is acculturative stress. Acculturative stress can be defined as the psychological impact of adaptation to a new culture [25]. For Hispanics who move to the U.S., there are a number of significant stressors (i.e., language conflict, family acculturation conflicts, perceived discrimination) that can be pervasive, intense, and lifelong due to acculturation. For example, rapid acculturation to the US among youth may lead to the adoption of unhealthy behaviors through increasing inter-generational stress within families (i.e., family acculturation conflicts), and subsequent loss of Hispanic/Latino customs and behaviors [26]. Thus, acculturative stress may play a significant role influencing health outcomes among Hispanic/Latino families.

The Hispanic/Latino family is a vital source of support, comprised of interdependent relationships that may cultivate cultural values and discourage family dysfunction $[27,28]$. This is important as youth in more dysfunctional families engage in unhealthier behaviors [29]. However, the role of family functioning in influencing SSB consumption, moderated by acculturation, remains an untested hypothesis.

This study aimed to examine the associations of acculturative stress and family functioning with SSB consumption by acculturation status among U.S. Hispanic/ Latino youth. We examined such associations under the assumption that on average, youth experience more rapid acculturation attainment, as well as youth adopt certain acculturation statuses on the basis of characteristics and experiences that are heterogenous across groups. We hypothesized that Hispanic/Latino youths' SSB consumption would differ by acculturation status and that acculturative stress and family functioning would be associated with Hispanic/Latino youth' SSB consumption across acculturation statuses.

\section{Methods}

\section{Data source and study sample}

This study used data from Hispanic Community Children's Health Study/Study of Latino Youth (SOL Youth) ancillary study to the Hispanic Community Health Study/Study of Latinos (HCHS/SOL) study, which is a comprehensive longitudinal multicenter community-based cohort study of Hispanic/Latino adults in the United States [30]. Between 2008 and 2011, 16,415 U.S. Hispanic/Latino adults were recruited from a random sample of households in four communities (i.e., Bronx, NY; Chicago, IL; Miami, FL; and San Diego, CA).

Between 2012 and 2014, SOL Youth recruited 1465 youth aged 8-16 years whose parent or caregiver was part of HCHS/SOL [31, 32]. For these analyses, we included youth with complete and valid SSB consumption data, as well as complete and valid data for family functioning, socio-cultural factors, and demographics. Study questionnaires to collect these data were administered 
by bilingual staff and in the youth language of preference. We excluded 210 cases due to incomplete data across key study variables. The overarching goal of the SOL-Youth study was to evaluate the influence of youth acculturation, effects of intergenerational influence in acculturation between youth and parents, parenting practices, as well as psychosocial functioning on cardiometabolic risk in Hispanic/Latino youth [31, 32]. SOLYouth had a cross-sectional design and included children who were assented to participate through voluntarily written informed consent. The study was conducted with approval of the Institutional Review Board at all study institutions, including the HCHS/SOL coordinating center and laboratory.

\section{Dependent measures}

\section{SSB consumption}

SSB consumption was measured through two 24-h dietary recalls from each child, with parental assistance if necessary. The initial dietary recall was conducted in person and the second dietary recall was conducted by telephone within a month of the first assessment. The Nutrition Data System for Research (NDSR) software was used to collect the data and to categorize SSB consumed into the following groups: (a) sugar sweetened beverages; (b) 100\% fruit juice; (c) sweetened soft drinks; and (d) sweetened fruit drinks. The average frequency of servings per day (8 fluid ounces) across beverage categories was used as the outcome measure.

\section{Independent measures \\ Acculturation}

Hispanic/Latino youth' acculturation status was measured using the Acculturation, Habits, and Interests Multicultural Scale for Adolescents (AHIMSA) [33]. Scale scores ranged from 1 to 8 . Under the assumption that acculturation itself is a dynamic process and that AHIMSA contains correlated sub-scales, youth were grouped into categories based on their acculturation status: Both Countries Orientation (Integration), United States orientation (Assimilation), orientation to other or neither country (Marginalization or Separation), and Unclassified. This categorical variable was used as grouping variable for descriptive purposes as well as the moderator in the final analytical output.

\section{Acculturative stress}

Hispanic/Latino youth' acculturative stress was measured using the Acculturative Stress Index [34] through three indicators: language conflict, family acculturation conflicts, and discrimination. To denote acculturative stress in our study, we developed a latent construct of acculturative stress composed of the three indicators aforementioned. This approach was used given that it accounts for measurement error, and tests whether these sub-domains represent well the higher construct (acculturative stress). Each indicator used a 5-point scale and, collectively, showed good compatibility to denote acculturative stress as a latent construct $(a>0.61 ; r>0.32$; $b>0.41)$.

\section{Family functioning}

Hispanic/Latino youths' family functioning was measured using the 12-item General Functioning subscale of the McMaster Family Assessment Device, which measures overall health of the family [35]. Example of statements include: "Planning family activities is difficult because we misunderstand each other;" "We cannot talk to each other about the sadness we feel;" "Making decisions is a problem for our family." To denote family functioning in our study, the average of all of the items was used. Family functioning scores ranged from 1 to 4 , with greater scores indicating poorer family functioning.

\section{Covariates}

Demographic characteristics included as covariates in all models were youth age in years (8-16 years old), youth' sex (male or female), birthplace (mainland U.S. or outside U.S.), parental educational attainment (less than high school, high School/GED, four-year college or university or more), family income (less than $\$ 20,000$, $\$ 20,000-\$ 50,000$, greater than $\$ 50,000)$, and household composition (continuous). The Healthy Eating Index (HEI-2010) was also used as a covariate to represent overall diet quality [36].

\section{Statistical analysis}

We used descriptive statistics to summarize demographic characteristics and key study variables (dependent and independent variables). Multi-group path regression models were used to test our study hypotheses using Structural Equation Modelling (SEM). Hispanic/Latino youth' SSB consumption was used as the dependent variable in all models. Hispanic/Latino youth' acculturative stress and family functioning were used as independent variables on SSB consumption to test our main hypothesis. Hispanic/Latino youth' acculturation status was used as the categorical grouping variable. Hispanic/Latino youth' age and sex were used as covariates in all models. We used 0.05 as the significance level. Model fit was assessed using the root mean square error of approximation (RMSEA), standardized root mean square residual (SRMR), and the Comparative Fit Index (CFI) [33]. In order for our models to attain good fit to the data, the following criteria had to be met: RMSEA ( $\leq 0.08)$, SRMR $(\leq 0.10)$, and CFI $(\geq 0.90)$ [37]. Missing data was handled using listwise deletion. Statistical software STATA 14 was used to perform all 
analyses while accounting for the complex survey design, sampling weights, and clustered nature of the data.

\section{Results}

Demographic characteristics are summarized in Table 1. The majority of children were 8-12 years old (57.5\%), and males and females were equally represented. A vast majority were born in the mainland United States (77.5\%), and half had low family income (52.9\%). Most youth were categorized as integrated (46.0\%), followed by assimilated (42.5\%), and lastly marginalized or separated (11.3\%). Overall, youth consumed approximately one SSB per day (0.96 servings/day), although marginalized or separated youth consumed slightly less SSB compared to youth with a stronger U.S. orientation (not statistically significant). Furthermore, fruit juice was the greatest contributor to overall SSB consumption across groups. Prior to testing the path regression models, ANOVA tests indicated mean SSB intake was statistically different across acculturation groups $(F(2,1301)=$ 3.43, $p=0.04)$ in unadjusted models. Chi-square tests also indicated statistical differences across acculturation groups in family functioning $\left(X^{2}=15.30, p<0.01\right)$.

Results from the path regression models are presented in Table 2. HEI and other demographic factors were excluded from models due to lack of preliminary correlation with key study variables $(r<0.20)$ and potential collinearity. Only youth' age, sex, and study site were the key covariates included in our final models. When controlling for age, sex, and study site, acculturative stress $(\beta=-0.13, p=0.01)$ was inversely associated with SSB among youth classified as assimilated, and poor family functioning ( $\beta=0.11, p=0.07)$ was only marginally associated with SSB consumption among youth classified as assimilated but not among youth classified as integrated, marginalized/separated, or unclassified.

\section{Discussion}

Findings from our study suggest limited support for our hypothesis that Hispanic/Latino youth' SSB consumption differs by acculturation status and for the hypothesis that family functioning would be associated with increased SSB consumption among Hispanic/Latino youth across acculturating groups. Acculturation differed across Hispanic/Latino youth in our sample, but no significant associations were found between acculturation categories and SSB consumption. There was a significant inverse association between acculturative stress and increased SSB consumption among assimilated Hispanic/Latino youth, but not in other acculturating groups.

Previous research suggests higher rates of SSB consumption have been generally observed among low income and racial/ethnic minority children and adults, particularly Hispanics/Latinos, compared with their higher income and non-Hispanic white counterparts [15]. Past research also suggests that youth of Hispanic/ Latino heritage experience greater risk of obesity-related outcomes, including diet [38, 39]. In our study, we found a significant relationship between acculturative stress and lower SSB consumption among youth classified as assimilated. On average, assimilated youth had greater acculturation conflicts compared to their counterparts with differing acculturation status. Whether related to diet or not, these conflicts could be further studied in their role limiting SSB consumption among assimilated youth. Another potential reason for this finding may be that assimilated Hispanic/Latino youth are more susceptible to being influenced by 'diet culture.' [40] Diet culture is a contemporary concept in which individuals adopt specific diets aiming at prioritizing or restricting consumption of foods based on broad societal standards. Future research should assess the implications for younger Hispanic/Latinos, who could be the most susceptible sub-group towards exposure to sport-based dietary marketing and potential restrictive eating patterns [41].

The Hispanic/Latino family is a vital context that shapes youth' health behaviors [42-46]. In our study, poor family functioning was marginally associated with greater SSB consumption among assimilated Hispanic/ Latino youth (not statistically significant). Additional research is needed to assess whether family (dys)function could be perceived as a stressor to assimilated Hispanic/ Latino youth who may turn to unhealthy energy-balance behaviors as a coping mechanism [43]. Implications for older Latino youth who are assimilated to the U.S. should also be considered in future research within the context of this relationship, as they may be most susceptible to increased SSB consumption in poor functioning family contexts [39]. Should the family structure, through functioning and cohesion, shape beverage consumption among Hispanic/Latino children, families may need to build capacity and stronger ties to create more healthful home and family environments especially under challenging circumstances [27-29, 44].

This study has strengths worth mentioning. Sample size for the study population is representative of some Hispanic/Latino urban communities in the U.S with diverse countries of origin. In addition, validated measures were used to assess all key constructs in the analyses, including SSB consumption assessed with two 24-h recalls used to capture culturally-relevant beverages in this population as our study was collecting data [47]. Lastly, by accounting for acculturation as a grouping variable for assessing effect modification, rather than a predicting variable within the context of a cross-sectional study, we maximized the potential for examining acculturation in a more nuanced way in combination with other sociocultural determinants of health (i.e., acculturative stress, family functioning). 
Table 1 Summary of characteristics of Hispanic/Latino youth, $n=1465$

\begin{tabular}{|c|c|c|c|c|c|}
\hline & $\begin{array}{l}\text { Total Sample } \\
(n=1465)\end{array}$ & $\begin{array}{l}\text { a Integrated } \\
(n=674)\end{array}$ & $\begin{array}{l}{ }^{\mathrm{b}} \text { Assimilated } \\
(n=495)\end{array}$ & $\begin{array}{l}\text { 'Separated or } \\
\text { marginalized }(n=92)\end{array}$ & $\begin{array}{l}{ }^{\mathrm{d}} \text { Unclassified } \\
(n=204)\end{array}$ \\
\hline SSB consumption (servings/day) & $0.96(0.75)$ & $0.98(0.80)$ & $0.98(0.72)$ & $0.90(0.62)$ & $0.85(0.62)$ \\
\hline Soft drinks & $1.14(1.07)$ & $1.19(1.14)$ & $1.15(1.03)$ & $1.10(0.92)$ & $0.98(0.90)$ \\
\hline Fruit juice & $1.79(1.35)$ & $1.80(1.41)$ & $1.83(1.29)$ & $1.66(1.09)$ & $1.66(1.29)$ \\
\hline Other beverages & $0.46(0.49)$ & $0.47(0.52)$ & $0.46(0.46)$ & $0.43(0.39)$ & $0.41(0.42)$ \\
\hline Family functioning & $1.93(0.43)$ & $1.88(0.42)$ & $1.99(0.44)$ & $1.99(0.46)$ & $1.93(0.41)$ \\
\hline \multicolumn{6}{|l|}{ Acculturation (AHIMSA scores) } \\
\hline antegrated & $3.56(2.14)$ & $5.47(1.15)$ & $1.60(1.09)$ & $1.32(0.99)$ & $3.03(1.15)$ \\
\hline${ }^{\mathrm{b}}$ Assimilated & $2.99(1.96)$ & $1.57(1.02)$ & $5.17(1.18)$ & $1.54(0.94)$ & $3.10(1.08)$ \\
\hline 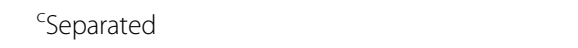 & $1.19(1.38)$ & $0.78(0.89)$ & $0.98(0.94)$ & $4.46(1.66)$ & $1.58(1.47)$ \\
\hline${ }^{c}$ Marginalized & $0.21(0.57)$ & $0.15(0.43)$ & $0.22(0.51)$ & $0.61(1.15)$ & $0.24(0.64)$ \\
\hline \multicolumn{6}{|l|}{ Age group } \\
\hline $8-12$ years old & $57.5 \%$ & $46.5 \%$ & $68.5 \%$ & $60.9 \%$ & $66.2 \%$ \\
\hline 13-16 years old & $42.4 \%$ & $53.5 \%$ & $31.5 \%$ & $39.1 \%$ & $33.8 \%$ \\
\hline \multicolumn{6}{|l|}{ Sex } \\
\hline Female & $49.6 \%$ & $52.9 \%$ & $47.6 \%$ & $47.8 \%$ & $49.5 \%$ \\
\hline Male & $50.3 \%$ & $47.0 \%$ & $52.3 \%$ & $52.1 \%$ & $50.4 \%$ \\
\hline \multicolumn{6}{|l|}{ Birthplace } \\
\hline U.S. Mainland & $77.5 \%$ & $73.2 \%$ & $83.6 \%$ & $71.9 \%$ & $76.4 \%$ \\
\hline Outside of the U.S. Mainland & $22.4 \%$ & $26.7 \%$ & $15.3 \%$ & $28.0 \%$ & $22.0 \%$ \\
\hline \multicolumn{6}{|l|}{ Parental education } \\
\hline Less than high school & $38.6 \%$ & $39.3 \%$ & $38.1 \%$ & $47.8 \%$ & $32.3 \%$ \\
\hline High school/GED & $28.5 \%$ & $26.8 \%$ & $29.4 \%$ & $25.0 \%$ & $32.8 \%$ \\
\hline Four-yr college/university or more & $32.8 \%$ & $33.5 \%$ & $32.1 \%$ & $27.1 \%$ & $34.3 \%$ \\
\hline \multicolumn{6}{|l|}{ Family income } \\
\hline Less than $\$ 20,000$ & $52.9 \%$ & $50.0 \%$ & $51.0 \%$ & $61.9 \%$ & $49.0 \%$ \\
\hline$\$ 20,000-\$ 50,000$ & $32.2 \%$ & $32.9 \%$ & $30.3 \%$ & $26.0 \%$ & $29.9 \%$ \\
\hline Greater than $\$ 50,000$ & $14.8 \%$ & $13.7 \%$ & $15.4 \%$ & $09.7 \%$ & $16.6 \%$ \\
\hline Household composition (number of members) & $4.28(1.42)$ & $4.29(1.44)$ & $4.24(1.37)$ & $4.38(1.52)$ & $4.30(1.40)$ \\
\hline \multicolumn{6}{|l|}{ Acculturative stress } \\
\hline Acculturation conflicts & $1.77(0.78)$ & $1.62(0.69)$ & $1.92(0.82)$ & $1.85(0.79)$ & $1.88(0.84)$ \\
\hline Perceived discrimination & $1.47(0.70)$ & $1.34(0.55)$ & $1.57(0.79)$ & $1.75(0.92)$ & $1.50(0.76)$ \\
\hline Language conflicts & $1.58(0.86)$ & $1.43(0.72)$ & $1.64(0.92)$ & $2.20(1.05)$ & $1.65(0.88)$ \\
\hline Healthy Eating Index (2010-HEI) & $53.9(12.8)$ & $54.0(13.0)$ & $52.8(12.6)$ & $54.5(12.7)$ & $54.4(12.5)$ \\
\hline
\end{tabular}

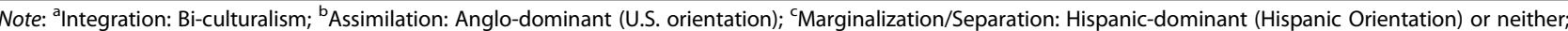
${ }^{\mathrm{d}}$ Unclassified: Acculturation status unknown

Data shown as mean (SD) or percent

Family functioning score ranged from 1 to 4 , with greater scores indicating poorer family functioning

AHIMSA score ranged from 1 to 8 , with greater scores indicating greater acculturation for each category

GED: General Education Diploma

Acculturative Stress: The psychological impact of adaptation to a new culture. For Hispanics who come to the U.S., there are a number of significant stressors that are likely to be pervasive, intense, and lifelong due to acculturation. Each domain of the acculturative stress scale ranges from 1 to 5 , with 5 more indicative of stress

Healthy Eating Index (2010-HEI) score ranges from 0 to 100 and is a measure of diet quality used to assess compliance with the 2010 U.S. Dietary Guidelines for Americans and monitor dietary changes; higher scores indicating greater consistency of the diet

Limitations in the study include the cross-sectional and self-reported nature of the overall data, which could introduce error to the inferences made. Thus, we are unable to make causal inferences, but rather report on the cross-sectional links (or lack thereof) and encourage future research that can elucidate the potential causal pathways (i.e., mediating factors at the family, home, and policy levels). For instance, children's SSB consumption 


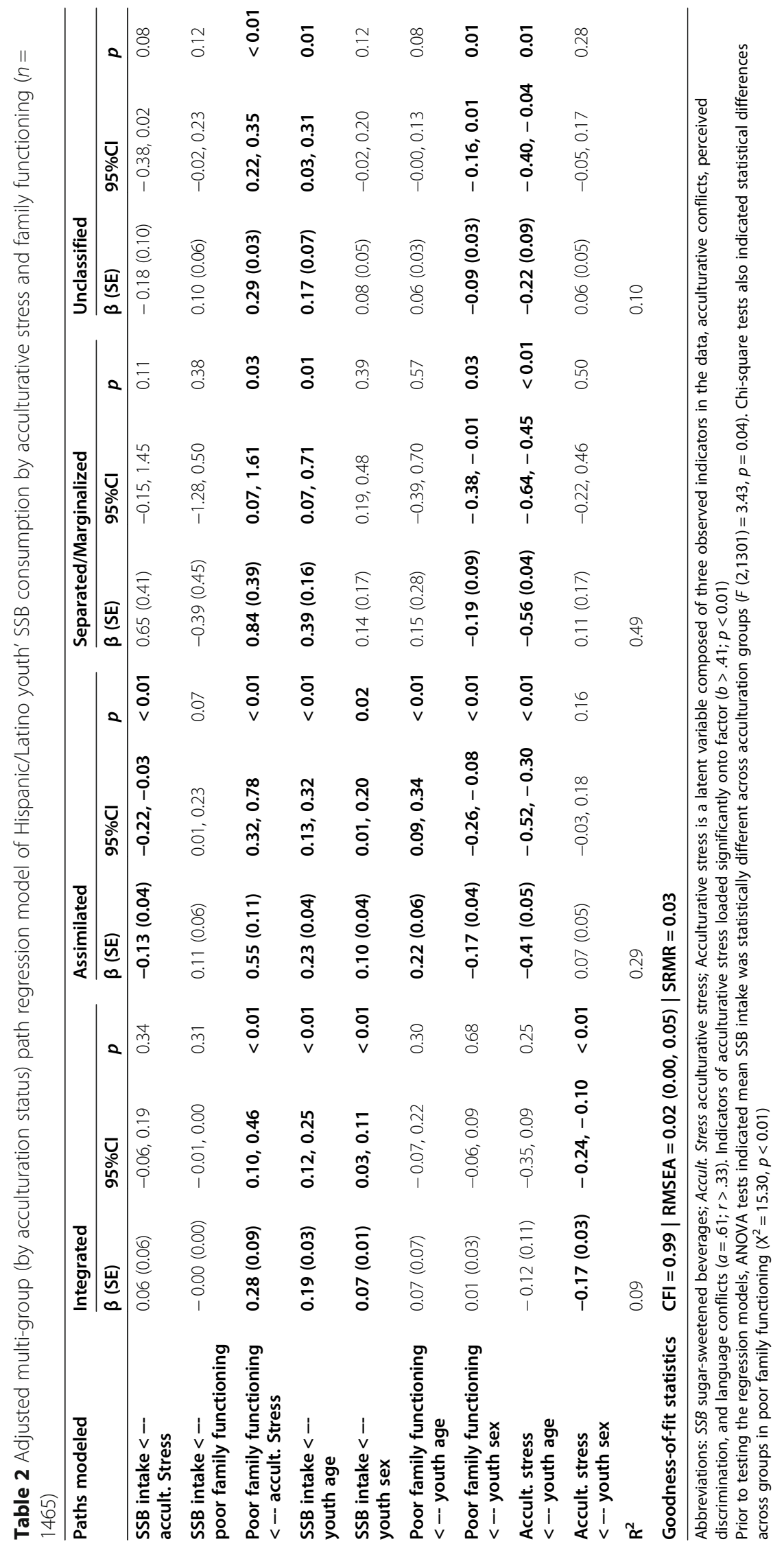


may be influenced by acculturative factors through parents' behaviors, practices, and their role shaping the home environment [48-51]. Also, we did not test for differences across Hispanic/Latino heritages due to small sample sizes, although studies among Hispanic/Latino adults have shown differences in acculturation and in SSB by heritage [21, 52]. Generalizability may be limited because some Hispanic/Latino youth may be underrepresented in relation to the geographic location of study recruitment. Lastly, future research is warranted to longitudinally assess acculturation as a process among Hispanic/Latino youth from diverse communities, as well as psychological and familial factors influencing links with dietary behaviors.

\section{Conclusion}

In conclusion, there is an inverse association between acculturative stress and SSB consumption, as well as a marginal but insignificant association between family functioning and SSB consumption among assimilated Hispanic/Latino youth. These preliminary results may support further research to inform preventive familybased approaches to help improve diet quality among Hispanic/Latino children by dissuading SSB intake. Future studies should assess these important interconnections more rigorously and longitudinally, accounting for theoretically relevant covariates. Specifically, examining the interplay between key cultural (i.e., acculturation), familial, and behavioral (i.e., dietary behaviors) risk factors of obesity while accounting for racial and ethnic-specific characteristics could inform future obesity research and programming tailored to minority groups disproportionally affected by obesity.

\section{Abbreviations}

SSB: Sugar-sweetened beverages; HCHS/SOL: Hispanic Community Children's Health Study/Study of Latino; NDSR: Nutrition Data System for Research; AHIMSA: Acculturation, Habits, and Interests Multicultural Scale for Adolescents; HEl-2010: Healthy Eating Index; SEM: Structural Equation Modelling

\section{Acknowledgements \\ The authors would like to thank the participants in this larger study who generously agreed to participate and provided study data. The authors also extend their gratitude towards members of the larger research study, especially to members who contributed to significant discussion within the HCHS/SOL Dietary Special Interest Group meetings. The authors are solely responsible for this study and its content does not necessarily represent the official views of the NHLBI or the $\mathrm{NIH}$.}

\section{Authors' contributions}

RF conceptualized the study, analyzed the data, and wrote the manuscript. JM supported the development of study from initial stage to subsequent significant revisions. CRI, KMP, ACM, LCG, DS-A, AMD, MD, and LVH contributed to the study concept and the revision of the manuscript; and all authors: read and approved the final manuscript.

\section{Funding}

The Hispanic Community Health Study/Study of Latinos was funded through collaborative contracts from the National Heart, Lung, and Blood Institute (NHLBI) to the University of North Carolina (N01-HC65233), University of
Miami (N01-HC65234), Albert Einstein College of Medicine (N01-HC65235), Northwestern University (N01-HC65236), and San Diego State University (N01-HC65237). At the earlier stages of this study, RF received support through the NIH Training Grant Postdoctoral Fellowship (T32DK007703). The funders did not have a role in the development of this study.

\section{Availability of data and materials}

The datasets generated and/or analyzed during the current are available in the National Institutes of Health (NIH), National Heart, Lung, Blood Institute (NHLBI), Biologic Specimen and Data Repository Information Coordinating Center, [https://biolincc.nhlbi.nih.gov/studies/hchssol/].

\section{Ethics approval and consent to participate}

This study included children who were assented to participate by parents through voluntarily written informed consent. The study was conducted with approval of the Institutional Review Board at San Diego State University, University of Illinois at Chicago, Albert Einstein College of Medicine in the Bronx area of New York City, and the University of Miami, as well as the $\mathrm{HCHS} / \mathrm{SOL}$ coordinating center and laboratory at the University of North Carolina in Chapel Hill.

\section{Consent for publication}

Not applicable.

\section{Competing interests}

The authors declare that they have no competing interests.

\section{Author details}

'Division of Nutritional Sciences, College of Human Ecology, Cornell University, Ithaca, NY, USA. ${ }^{2}$ Department of Epidemiology and Population Health, Albert Einstein College of Medicine, New York, NY, USA. ${ }^{3}$ Department of Social Medicine, University of North Carolina School of Medicine, Chapel Hill, NC, USA. ${ }^{4}$ School of Exercise and Nutritional Sciences, San Diego State University, San Diego, CA, USA. ${ }^{5}$ Department of Psychology, San Diego State University, San Diego, CA, USA. ${ }^{6}$ Department of Biostatistics, Gillings School of Global Health, University of North Carolina, Chapel Hill, NC, USA.

${ }^{7}$ Department of Pediatrics, Mailman Center for Child Development, University of Miami, Miami, FL, USA. ${ }^{8}$ Department of Preventive Medicine, Feinberg School of Medicine, Northwestern University, Chicago, IL, USA. ${ }^{9}$ Department of Nutrition, Harvard T.H. Chan School of Public Health, Harvard University, Boston, MA, USA.

Received: 13 April 2020 Accepted: 8 October 2020

Published online: 14 October 2020

\section{References}

1. Hu FB. Resolved: there is sufficient scientific evidence that decreasing sugarsweetened beverage consumption will reduce the prevalence of obesity and obesity-related diseases. Obes Rev. 2013;14(8):606-19 [PubMed: 23763695].

2. Malik VS, Schulze MB, Hu FB. Intake of sugar-sweetened beverages and weight gain: a systematic review. Am J Clin Nutr. 2006;84(2):274-88.

3. Malik VS, Pan A, Willett WC, Hu FB. Sugar-sweetened beverages and weight gain in children and adults: a systematic review and meta-analysis. Am J Clin Nutr. 2013;98(4):1084-102.

4. Popkin BM, Hawkes C. Sweetening of the global diet, particularly beverages: patterns, trends, and policy responses. Lancet Diabetes Endocrinology. 2016; 4(2):174-86

5. Singh GM, Micha R, Khatibzadeh S, Lim S, Ezzati M, Mozaffarian D. Estimated Global, Regional, and National Disease Burdens Related to Sugar-Sweetened Beverage Consumption in 2010. Circulation. 2015;114:010636.

6. Te Morenga L, Mallard S, Mann J. Dietary sugars and body weight: systematic review and meta-analyses of randomised controlled trials and cohort studies. Br Med J. 2013;346:e7492 [PubMed: 23321486].

7. Te Morenga LA, Howatson AJ, Jones RM, Mann J. Dietary sugars and cardiometabolic risk: systematic review and meta-analyses of randomized controlled trials of the effects on blood pressure and lipids. Am J Clin Nutr. 2014;100(1):65-79 [PubMed: 24808490].

8. Vartanian $L R$, Schwartz MB, Brownell KD. Effects of soft drink consumption on nutrition and health: a systematic review and meta-analysis. Am J Public Health. 2007;97(4):667-75 [PubMed: 17329656]. 
9. Mourao DM, Bressan J, Campbell WW, Mattes RD. Effects of food form on appetite and energy intake in lean and obese young adults. International Journal of Obesity (London). 2007;31(11):1688-95.

10. Powell ES, Smith-Taillie LP, Popkin BM. Added sugars intake across the distribution of US children and adult consumers: 1977-2012. J Acad Nutr Diet. 2016;116(10):1543-50.

11. Harvard School of Public Health (HSPH). (2018). The Nutrition Source: Sugary Drinks. Retrieved from: https://www.hsph.harvard.edu/nutritionsource/ healthy-drinks/sugary-drinks/.

12. Flood-Obbagy JE, Rolls BJ. The effect of fruit in different forms on energy intake and satiety at a meal. Appetite. 2009;52(2):416-22 [PubMed: 19110020].

13. Ludwig, D. S., \& Ebbeling, C. B. (2018). The carbohydrate-insulin model of obesity: beyond "calories in, calories out". JAMA internal medicine.

14. Moreno LA, Rodríguez G, Fleta J, Bueno-Lozano M, Lázaro A, Bueno G. Trends of dietary habits in adolescents. Crit Rev Food Sci Nutr. 2010;50(2): 106-12.

15. Bleich SN, Vercammen KA, Koma JW, Li Z. Trends in beverage consumption among children and adults, 2003-2014. Obesity. 2018;26(2):432-41.

16. Centers for Disease Control and Prevention (CDC). "The Health Effects of Overweight and Obesity." Available at: https://www.cdc.gov/healthyweight/ effects/index.html.

17. Akintayo DMO. Obesity and Hispanic children: the influence of culture on obesity. J Res Education. 2013;2:9.

18. Berry JW. Immigration, acculturation, and adaptation. Appl Psychol. 1997; 46(1):5-34.

19. Evans A, Chow S, Jennings R, Dave J, Scoblick K, Sterba KR, Loyo J. Traditional foods and practices of Spanish-speaking Latina mothers influence the home food environment: implications for future interventions. J Am Diet Assoc. 2011;111(7):1031-8.

20. Unger JB, Reynolds K, Shakib S, Spruijt-Metz D, Sun P, Johnson CA. Acculturation, physical activity, and fast-food consumption among AsianAmerican and Hispanic adolescents. J Community Health. 2004;29(6):467-81.

21. Park S, Blanck HM, Dooyema CA, Ayala GX. Association between sugarsweetened beverage intake and proxies of acculturation among US Hispanic and non-Hispanic white adults. Am J Health Promot. 2016;30(5): 357-64

22. Arandia G, Sotres-Alvarez D, Siega-Riz AM, Arredondo EM, Carnethon MR, Delamater AM, Van Horn L. Associations between acculturation, ethnic identity, and diet quality among US Hispanic/Latino youth: findings from the HCHS/SOL youth study. Appetite. 2018.

23. Rudmin F. Constructs, measurements and models of acculturation and acculturative stress. Int J Intercult Relat. 2009;33(2):106-23.

24. Zambrana RE. Understanding Latino families: Scholarship, policy, and practice (Vol. 2). Sage; 1995.

25. Smart JF, Smart DW. Acculturative stress: the experience of the Hispanic immigrant. Couns Psychol. 1995;23(1):25-42.

26. Wagner KD, Ritt-Olson A, Chou CP, Pokhrel P, Duan L, Baezconde-Garbanati $L$, Unger JB. Associations between family structure, family functioning, and substance use among Hispanic/Latino adolescents. Psychol Addict Behav. 2010;24(1):98.

27. Rivera FI, Guarnaccia PJ, Mulvaney-Day N, Lin JY, Torres M, Alegría M. Family cohesion and its relationship to psychological distress among Latino groups. Hispanic J Behav Sci. 2008;30(3):357-78. https://doi.org/10.1177/ 0739986308318713.

28. Lorenzo-Blanco El, Unger JB, Baezconde-Garbanati L, Ritt-Olson A, Soto D. Acculturation, enculturation, and symptoms of depression in Hispanic youth: the roles of gender, Hispanic cultural values, and family functioning J Youth Adolesc. 2012;41(10):1350-65.

29. Abraído-Lanza AF, Echeverría SE, Flórez KR. Latino immigrants, acculturation, and health: promising new directions in research. Annu Rev Public Health. 2016:37:219-36.

30. United States Department of Health and Human Services (USDHHS). (2013). "Hispanic Comunity Health Study Data Book: A Report to Communities." Available at: https://www.nhlbi.nih.gov/files/docs/resources/NHLBIHCHSSOL-English-508.pdf.

31. Ayala GX, Carnethon M, Arredondo E, Delamater AM, Perreira K, Van Horn L, Isasi CR. Theoretical foundations of the study of Latino (SOL) youth: implications for obesity and cardiometabolic risk. Ann Epidemiol. 2014;24(1):36-43.

32. Isasi CR, Carnethon MR, Ayala GX, Arredondo E, Bangdiwala SI, Daviglus ML, Kaplan RC. The Hispanic community children's health study/study of Latino youth (SOL youth): design, objectives, and procedures. Ann Epidemiol. 2014; 24(1):29-35.

33. Unger JB, Gallaher P, Shakib S, Ritt-Olson A, Palmer PH, Johnson CA. The AHIMSA acculturation scale: a new measure of acculturation for adolescents in a multicultural society. J Early Adolesc. 2002;22(3):225-51.

34. Noh S, Avison WR. Asian immigrants and the stress process: a study of Koreans in Canada. J Health Soc Behav. 1996;37:192-206.

35. Miller IW, Epstein NB, Bishop DS, Keitner Gl. The McMaster family assessment device: reliability and validity. J Marital Fam Ther. 1985;11(4): 345-56

36. Guenther PM, Casavale KO, Reedy J, Kirkpatrick SI, Hiza HA, Kuczynski KJ et al. Update of the healthy eating index: HEI-2010. J Acad Nutr Diet. 2013; 113(4):569-80.

37. $\mathrm{Hu}$ L, Bentler PM. Cutoff criteria for fit indexes in covariance structure analysis: conventional criteria versus new alternatives. Struct Equ Modeling. 1999;6(1):1-55. https://doi.org/10.1080/10705519909540118.

38. Gordon-Larsen P, Harris KM, Ward DS, Popkin BM. Acculturation and overweight-related behaviors among Hispanic immigrants to the US: the National Longitudinal Study of adolescent health. Soc Sci Med. 2003;57(11): 2023-34.

39. Wen CKF, Hsieh S, Huh J, Martinez LC, Davis JN, Weigensberg M, SpruijtMetz D. The role of assimilating to the US culture and the relationship between neighborhood ethnic composition and dietary intake among Hispanic youth. J Racial Ethn Health Disparities. 2017:4(5):904-10.

40. Coggins NM, Coggins K. I'm not on a diet: culture, Health and Healing: Sunstone Press; 2010.

41. Cordero ED, Gutierrez A. Weight-related eating among less-acculturated Latina college students. J Immigr Minor Health. 2016;18(5):1032-7.

42. Birch LL, Davison KK. Family environmental factors influencing the developing behavioral controls of food intake and childhood overweight. Pediatr Clin. 2001 Aug 1;48(4):893-907.

43. Davison KK, Birch LL. Childhood overweight: a contextual model and recommendations for future research. Obes Rev. 2001 Aug 1;2(3):159-71.

44. Lebron CN, Lee TK, Park SE, St George SM, Messiah SE, Prado G. Effects of parent-adolescent reported family functioning discrepancy on physical activity and diet among Hispanic youth. J Fam Psychol. 2018;32(3):333.

45. Harrison K, Bost KK, McBride BA, Donovan SM, Grigsby-Toussaint DS, Kim J, et al. Toward a developmental conceptualization of contributors to overweight and obesity in childhood: the six-Cs model. Child Dev Perspect. 2011;5(1):50-8.

46. Halliday JA, Palma CL, Mellor D, Green J, Renzaho AMN. The relationship between family functioning and child and adolescent overweight and obesity: a systematic review. Int J Obes. 2014;38(4):480.

47. Subar AF, Freedman LS, Tooze JA, Kirkpatrick SI, Boushey C, Neuhouser ML, et al. Addressing current criticism regarding the value of self-report dietary data. J Nutr. 2015;145(12):2639-45.

48. van Ansem WJ, van Lenthe FJ, Schrijvers $C T$, Rodenburg G, van de Mheen D. Socio-economic inequalities in children's snack consumption and sugarsweetened beverage consumption: the contribution of home environmental factors. Br J Nutr. 2014;112(3):467-76.

49. Luszczynska A, de Wit JB, de Vet E, Januszewicz A, Liszewska N, Johnson F, Stok FM. At-home environment, out-of-home environment, snacks and sweetened beverages intake in preadolescence, early and mid-adolescence: the interplay between environment and self-regulation. J Youth Adolesc. 2013;42(12):1873-83.

50. Johansen $\mathrm{CM}$. The mediating role of social and contextual factors on the relationship between acculturation and health behaviors among Latino adolescents Doctoral dissertation: The Claremont Graduate University); 2019.

51. Davis RE, Cole SM, Reyes LI, McKenney-Shubert SJ, Peterson KE. "It hurts a Latina when they tell us anything about our children": implications of Mexican-origin mothers' maternal identities, aspirations, and attitudes about cultural transmission for childhood obesity prevention. Child Obes. 2015; 11(5):608-15.

52. Mattei J, Sotres-Alvarez D, Daviglus ML, Gallo LC, Gellman M, Hu FB, et al. Diet quality and its association with cardiometabolic risk factors vary by Hispanic and Latino ethnic background in the Hispanic community health study/study of Latinos. J Nutr. 2016;146(10):2035-44.

\section{Publisher's Note}

Springer Nature remains neutral with regard to jurisdictional claims in published maps and institutional affiliations. 